\title{
Marie-Françoise Baslez (dir.), Chrétiens persécuteurs. Destructions, exclusions, violences religieuses au $\mathrm{IV}^{\mathrm{e}}$ siècle
}

Paris, Albin Michel, coll. « Bibliothèque Albin Michel Histoire », 2014, $458 \mathrm{p}$.

\section{Anna van den Kerchove}

\section{(2) OpenEdition} Journals

Édition électronique

URL : http://journals.openedition.org/assr/28182

DOI : $10.4000 /$ assr.28182

ISSN : $1777-5825$

Éditeur

Éditions de l'EHESS

Édition imprimée

Date de publication : 31 décembre 2016

Pagination : 262

ISSN : 0335-5985

Référence électronique

Anna van den Kerchove, « Marie-Françoise Baslez (dir.), Chrétiens persécuteurs. Destructions, exclusions, violences religieuses au ive siècle », Archives de sciences sociales des religions [En ligne], 176 | octobre-décembre 2016, mis en ligne le 17 juillet 2017, consulté le 24 septembre 2020. URL: http://journals.openedition.org/assr/28182 ; DOI : https://doi.org/10.4000/assr.28182 


\section{Marie-Françoise Baslez (dir.), Chrétiens persécuteurs. Destructions, exclusions, violences religieuses $\mathrm{au} \mathrm{IV}{ }^{\mathrm{e}}$ siècle}

Paris, Albin Michel, coll. « Bibliothèque Albin Michel Histoire », 2014, $458 \mathrm{p}$.

Anna van den Kerchove

\section{RÉFÉRENCE}

Marie-Françoise Baslez (dir.), Chrétiens persécuteurs. Destructions, exclusions, violences religieuses au Iv ${ }^{\mathrm{e}}$ siècle

Marie-Françoise Baslez a réuni plusieurs historiens des religions spécialistes de la fin de l'Antiquité autour de la thématique des "violences religieuses" au IV siècle et plus particulièrement autour de l'étude de l'attitude des chrétiens, qui de pratiquants d'un culte minoritaire et illégal deviennent membres d'un culte légal et peu à peu majoritaire. Bien que le point d'interrogation ne fasse pas partie du titre (pour des raisons principalement éditoriales), la question qui est à l'arrière-plan de l'ouvrage est de déterminer si les chrétiens, une fois reconnus et soutenus par le pouvoir impérial, deviennent à leur tour persécuteurs. Pour répondre à cette question implicite, plusieurs études de cas sont proposées à la demande de Baslez (p. 9), à partir desquelles cette dernière suggère en conclusion une réponse générale. Différents types de violence sont ainsi abordés, que ce soit les modalités pratiques de l'exercice de la violence (violence verbale et violence physique; violence sur les bâtiments et les ouvrages écrits, empêchant la diffusion des idées ou la pratique régulière d'un culte), les destinataires (polythéistes, juifs et tous ceux qui sont considérés comme déviants ou « hérétiques ») et les acteurs (législation impériale, moines, etc.). 
2 L'ouvrage est introduit par quelques mots de la coordinatrice (p. 7-10) qui revient rapidement sur le changement intervenu au début $\mathrm{du}_{\mathrm{IV}}{ }^{\mathrm{e}}$ siècle en termes de relation des chrétiens aux autres et qui explicite la méthodologie utilisée dans l'ouvrage : les études de cas. Le lecteur pourra regretter l'absence de définition de concepts clés comme " persécution », «tolérance » et « intolérance». De plus, il pourra être surpris de lire le nom de Pilate quand il est question des gouverneurs de province dont les chrétiens sont les victimes et d'apprendre que les chrétiens sont persécutés depuis la Passion du Christ (p. 7). Peut-on parler de chrétiens pour une période aussi haute ? Et s'agit-il d'une persécution?

3 Cette brève introduction est suivie par une utile mise en perspective historiographique due à Béatrice Caseau: "Christianisation et violence religieuse: le débat historiographique»(p.11-36). Après un rappel des positions contradictoires sur l'appréciation d'une part de la violence au $\mathrm{IV}^{\mathrm{e}}$ siècle (avec une comparaison avec les siècles qui précèdent le $\mathrm{IV}^{\mathrm{e}}$ siècle), d'autre part du lien entre monothéisme et violence, l'auteur met en garde contre le prisme des sources et rappelle, à la suite de David Frankfurter, l'importance de la prise en compte du genre narratif de plusieurs d'entre elles.

4 Plusieurs contributions concernent la législation impériale. Dans « Le devoir religieux des empereurs : de la tolérance à la répression » (p.37-62), Pierre Maraval fait une distinction entre la législation antérieure à Théodose et la législation postérieure. Il considère qu'il y a une absence de contrainte de la part de Constantin, dans sa volonté de maintenir l'ordre public, et il met en avant le caractère novateur de Théodose qui énonce dans ses lois ce qu'il faut croire. Le lecteur pourra peut-être regretter que l'emploi des termes comme tolérance ou intolérance ne soit pas plus justifié. Laurent Guichard poursuit cette étude de la législation en s'interrogeant sur son caractère oppressif : «Des lois oppressives? La législation des empereurs chrétiens de haereticis et paganis » (p. 63-89). L'auteur conclut par l'affirmative, distinguant les lois visant les " hérétiques ", plus oppressives que celles visant les "païens "; toutefois, il modère ensuite son propos, puisqu'il estime que les lois du IV siècle n'ont pas été aussi persécutrices que les lois antérieures à 313 , du fait de l'absence de grands procès et d'exécutions.

5 Une telle étude ne peut se passer d'une approche lexicale, et en particulier de la manière dont l'« autre » est qualifié : « hérétique », « païen », qualificatifs tous négatifs. D'autres termes sont aussi utilisés; ainsi à propos des donatistes pour parler de leur « écart » par rapport aux autres chrétiens. C'est le cœur du propos de François-Xavier Romanacce : «Étude de la catégorisation dans la répression religieuse : le donatisme, parti, schisme, hérésie » (p. 91-138). Il s'intéresse au cas particulier de l'Église donatiste en Afrique du Nord et à la manière dont le vocabulaire évolue (schisme puis hérésie), avec le tournant de la législation impériale d'Honorius en 405.

6 L'article suivant poursuit l'étude de la législation impériale, en s'intéressant cette fois au troisième "adversaire religieux » : après les "païens ", après les " hérétiques ", les juifs. Capucine Nemo-Pekelman s'interroge ainsi sur l'attitude impériale vis-à-vis des juifs : «Le législateur chrétien a-t-il persécuté les juifs?»(p. 138-162). Elle commence quelques pages très utiles sur l'évolution du mot persecutio sous la plume des penseurs chrétiens antiques qui adjoignent à ce terme les idées d'injustice et de cruauté, sur la plasticité actuelle de "persécution » et sur la manière dont l'historien peut lui aussi parler d'injustice et de cruauté et dont il intègre l'idée d'une persistance de la 
persécution. Elle fait également une distinction entre lois pénales et lois visant l'ensemble d'une population donnée (ici les juifs). Ces pages pourraient être lues comme introduction à plusieurs contributions du volume. Ensuite, l'auteur examine les lois portant sur les juifs et conclut que celles-ci font preuve d'une "sévérité moindre ", mais (et cela nous paraît à retenir) non pas du fait d'une volonté impériale, mais de l'action des juifs eux-mêmes « qui surent utiliser au mieux les voies de droit » (p. 162).

7 Sébastien Morlet poursuit la réflexion sur les relations entre chrétiens et juifs, quittant le domaine législatif pour le domaine littéraire. Dans «L'antijudaïsme chrétien au $\mathrm{IV}^{\mathrm{e}}$ siècle. À propos de quelques idées reçues » (p. 163-188), l'auteur revient sur le terme récent " antisémitisme » et sur les mots anciens, grecs et latins, "genos, genus ». Ces derniers sont très souvent traduits par « race», une traduction que l'auteur juge, à juste titre, «trompeuse » (p. 171). L'examen des écrits d'Eusèbe, de Jean Chrysostome ou d'Augustin témoigne d'un regard chrétien très divers; l'auteur note que les échanges persistent, même s'il y a aussi des heurts. Une réponse simple ne peut donc être faite pour ce qui concerne les relations des chrétiens avec les juifs au IV siècle.

8 La loi édicte, mais nous savons combien l'écart peut être grand entre ce qu'elle édicte et son application concrète. C'est ce qu'examine Bernadette Cabouret : «Application de la loi et initiatives individuelles : le témoignage de Libanios d'Antioche» (p. 189-219). Le point de départ est le discours de Libanios, La défense des sanctuaires, que l'auteur confronte à d'autres sources de l'époque, notamment pour ce qui concerne le rôle des évêques et des moines. Emmanuel Soler aborde «Les victimes des procès de 371-372 à Rome et à Antioche : comment furent liquidés les réseaux de théurges »(p. 221-254). Il montre les ressorts politiques et religieux à la fois du groupe des partisans de Julien et de la répression qui a touché ces derniers. Il revient sur l'idée selon laquelle tous les condamnés étaient théurges et sur celle d'une répression massive. Sur ce dernier point, sa réflexion est poursuivie par Jean Bouffartigue, malheureusement décédé avant la publication de l'ouvrage: "Après la mort de l'Apostat, qu'est devenu le réseau de Julien?» (p. 255-283). L'auteur, à travers des études de cas individuels, met en valeur la grande variété des sorts subis par les proches de Julien après la mort de ce dernier. L'étude de l'attitude des chrétiens vis-à-vis des polythéistes ne pouvait pas ne pas aborder la question d'Hypatie. C'est Clelia Martínez Maza qui s'en charge: «Une victime sans importance? La mort de la philosophe Hypatie» (p. 285-310). L'auteur rappelle les circonstances de la mort d'Hypatie qu'elle relie au contexte général des tensions entre le préfet et l'évêque, avant d'étudier la réaction du pouvoir impérial ou plutôt l'absence de réaction du pouvoir impérial.

9 Les deux contributions suivantes abordent la question de l'appropriation de l'espace, en étudiant les cas particuliers d'une part de synagogues, d'autre part de l'action d'un évêque "non-orthodoxe ». Dans " Des paroles aux actes. La destruction des synagogues et leur transformation en églises» (p.311-335), Pierluigi Lanfranchi confronte différentes sources dont l'historien dispose (témoignages littéraires, législation impériale et sources archéologiques) sur le devenir des synagogues. Il conclut à une évolution au cours $d u \mathrm{IV}^{\mathrm{e}}$ siècle et à un rapport complexe entre discours et réalité concrète. Il appelle à une mise en perspective plus générale, en comparant l'attitude vis-à-vis des juifs à celle envers les "païens » et les « hérétiques », et il propose l'idée d'une "purification de l'espace urbain » qui serait "pollué » par l'idolâtrie des nonchrétiens (p.330). Quant à Philippe Blaudeau, dans «Détruire pour construire une identité civique? L'œuvre de l'évêque homéousien Éleusios à Cyzique » (p. 337-360), il 
analyse le cas particulier d'un évêque «non-orthodoxe » qui cherche à construire dans l'espace une nouvelle identité pour sa communauté. L'auteur considère que cette entreprise ne relève pas en tant que telle de la contrainte, ni de la haine de l'autre.

Les trois dernières contributions reviennent sur les relations des chrétiens aux polythéistes et sur la responsabilité (ou non) des premiers dans la disparition de pratiques ou de statues cultuelles. Dans « Les chrétiens et les cultes à mystères dans les provinces hispaniques. Question de responsabilité et apport de l'archéologie» (p.361-394), Jaime Alvar s'interroge sur la responsabilité des chrétiens dans la disparition des cultes à mystères. Pour y répondre, il est important de savoir quand l'historien peut dater cette disparition. À partir de l'analyse de cas particuliers, l'auteur invite à plus de prudence et à éviter de faire des généralisations. Yann Le Bohec propose une brève communication sur «Les maisons de la cachette» (p. 395-402). Les découvertes de pièces fermées dans des villas à Carthage contenant de nombreuses statues et la datation de ces villas témoigneraient que des destructions eurent lieu dès le règne de Constantin. Christian R. Raschle s'intéresse aux oracles: "Mettre les religions en concurrence: la fin des oracles»(p.403-437). Il rappelle la lutte des chrétiens contre les oracles à la fois sur le plan philosophique et sur le plan législatif et il termine en considérant que le culte des martyrs se substitue aux oracles et qu'il est un facteur pour attirer les « païens".

11 Dans des « Conclusions » (p. 439-444), Marie-Françoise Baslez revient sur la persistance et l'acharnement qui définissent juridiquement la persécution, pour affirmer que celleci ne cadre pas avec la diversité des attitudes des chrétiens vis-à-vis des non-chrétiens ou des hérétiques. Cela amène l'auteur à considérer que " tout conduit à relativiser la responsabilité des chrétiens» (p.444). Elle répond ainsi négativement à la question posée (implicitement) dans le titre de l'ouvrage: si les chrétiens ont été persécutés avant Constantin, ils ne sont pas devenus persécuteurs après 313.

Les études de cas sont intéressantes, même si elles ne renouvellent pas toutes la recherche et que certaines proposent une synthèse de résultats déjà connus. L'ouvrage intéressera ainsi un large public de chercheurs et d'étudiants. Que l'on nous permette seulement d'émettre une réserve quant à la conclusion. Au vu des documents qui sont parvenus jusqu'à nous, il nous semble difficile d'émettre une conclusion générale et encore plus de comparer l'attitude des chrétiens vis-à-vis de l'« autre » à l'attitude des polythéistes face aux chrétiens avant Constantin; il n'est en effet pas sûr que les mêmes critères soient appliqués (notamment celui de la persistance, utilisé en conclusion) dans l'étude des «violences » de la part de polythéistes, puis de la part des chrétiens. 